Revue internationale de l'économie sociale

Recma

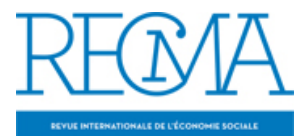

\title{
En hommage à Jacques Moreau (1927-2004)
}

\section{André Chomel}

Numéro 291, février 2004

URI : https://id.erudit.org/iderudit/1022114ar

DOI : https://doi.org/10.7202/1022114ar

Aller au sommaire du numéro

\section{Éditeur(s)}

Institut de l'économie sociale (IES)

\section{ISSN}

1626-1682 (imprimé)

2261-2599 (numérique)

Découvrir la revue

\section{Citer ce document}

Chomel, A. (2004). En hommage à Jacques Moreau (1927-2004). Revue internationale de l'économie sociale, (291), 6-8.

https://doi.org/10.7202/1022114ar d'utilisation que vous pouvez consulter en ligne.

https://apropos.erudit.org/fr/usagers/politique-dutilisation/ 


\section{EN HOMMAGE À JACQUES MOREAU (1927-2004)}

(1) Un ouvrage d'hommage à Jacques Moreau sera publié prochainement dans notre collection "Les cahiers de l'économie sociale ". 'équipe de la Recma est en deuil de Jacques Moreau, qui s’en est allé le 8 janvier ${ }^{(1)}$. L'hommage que nous lui devons, et auquel s'associeront nombre de lecteurs de la revue, sera acte de mémoire et expression d'une reconnaissance.

Chacun sait le rôle majeur qui a été le sien comme président-directeur général du groupe du Crédit coopératif. Son action à ce titre s'inscrit fortement dans l'histoire de l'invention de l'économie sociale au second XXe siècle. Mais la place réelle qui reviendra à Jacques Moreau dans cette histoire déborde singulièrement celle de l'éminent banquier de l'économie sociale qu'il aura été. L'ouvre qu'il laisse est aussi faite des initiatives qu'il a prises, des impulsions qu'il a données à l'animation de la recherche sur l'économie sociale et de ses propres contributions à la "rénovation de son concept", pour reprendre son expression dans le saisissant témoignage sur "L'évolution du Crédit coopératif de 1974 à 1992 " publié en 2001 dans la Recma; ce texte est cependant trop discret sur ce second volet de son œuvre, pourtant étroitement lié au premier.

Quelques temps forts sont donc à évoquer ici. Il y eut ainsi en 1984 la réanimation de la Revue des études coopératives, fondée en 1921 par Charles Gide. Celle-ci doit à Jacques Moreau d'exister encore au début du XXI siècle. La Rec avait cessé de paraitre en 1983, victime de la crise des coopératives de consommateurs. C'est la décision du président Moreau qui permit à la jeune Fondation du Crédit coopératif de s'aventurer à en reprendre l'édition malgré le pessimisme ambiant touchant les chances de l'opération. On connait la suite avec la belle vitalité de la Revue internationale de l'économie sociale d'aujourd'hui. Dans cette suite, Jacques Moreau donnera son appui au maintien de l'identité d'une revue à caractère scientifique associant chercheurs et acteurs de l'économie sociale, contre la tendance récurrente à la banalisation sous forme de magazine.

La relance de la revue avait été précédée d'une première initiative à longue portée pour une connaissance scientifique des organisations de l'économie sociale: ce fut la création, avec André Chadeau, de l'Association pour le développement de la documentation sur l'économie sociale (Addes), ouvrant de nouvelles pistes de recherches. Dans la foulée, une base de données sur l'économie sociale verra le jour au Crédit coopératif, Sydes, comblant le vide résultant de l'abandon de celle qui existait à l'Idec, entrainée dans la crise de la coopération de consommation.

Les instances de ces organes seront un creuset pour l'ouverture du champ et l'appel à de nouveaux chercheurs, favorisant le relais de la génération 
représentée par Henri Desroche et Claude Vienney, «les deux pôles de notre recherche » selon l'expression de J.-F. Draperi; l'un et l'autre s'investiront d'ailleurs activement dans les voies ainsi offertes. Jacques Moreau trouvera un jour dans cette proximité l'occasion d'une confrontation entre sa proposition de "l'économie sociale sans rivage " et le thème cher à Desroche $\mathrm{du}$ "désenclavement de l'économie sociale».

La coopération avec Henri Desroche et son Université coopérative internationale donnera lieu à un développement imprévu avec la coorganisation en mai 1984, au Crédit coopératif, d'un grand colloque sur le crédit au Sud et la coopération d'épargne et de crédit en Afrique (dont les travaux ont été publiés dans la Revue des études coopératives de mai 1985). Celui-ci se prolongera quelque temps après par le lancement, à l'initiative de la Fondation du Crédit coopératif, d'un réseau de coopératives d'épargne et de crédit pour les paysans du Mali sud, Kafo Jiginew, auquel le président et Mme Moreau rendront visite en 1999.

Bien d'autres initiatives suivront, et entre autres l'appui au Comité pour l'édition des œuvres de Charles Gide; Jacques Moreau en assumera la présidence pour son lancement en 1995.

Il y a enfin, ou surtout, ses contributions personnelles, articles et ouvrages, entre autres son Essai sur une politique de l'économie sociale et L'économie sociale face à l'ultralibéralisme. Sans doute Jacques Moreau y présente-t-il son propos plus comme un appel, ou une provocation au débat, que comme des propositions magistérielles. Ces textes n'en sont pas moins une invitation pressante à "réintroduire l'économie sociale dans le champ des idées". On reviendra sans doute dans le futur sur cette pensée vigoureuse et originale, entre appel à l'utopie pour stimuler l'imagination, réalisme des propositions pour une économie sociale voulue, insistance sur la position centrale du sociétariat dans les organisations.

Lautorité de cette pensée, conjuguée à celle du manager, a fait de Jacques Moreau un leader incontesté de l'économie sociale dans les dernières décennies du XXe siècle.

La vision qui a présidé à l'ensemble de son œuvre est celle d'un homme de conviction et de grande culture, exigeant et généreux, partageant sa foi dans un avenir d'économie plus humaine avec ceux qui, comme lui, vivaient le troisième secteur.

A son épouse, Mme Mireille Moreau, et à ses enfants, Hélène et Antoine, l'équipe de la Recma exprime sa respectueuse sympathie.

Pour la Recma, A.C. 


\section{Articles et ouvrages de Jacques Moreau, sélection}

\section{Ouvrages}

Pour une économie sociale sans rivages, Paris, Crédit coopératif, 1980.

Essai sur une politique de l'économie sociale, $d u$ troisième secteur vécu à un troisième secteur voulu, Ciem, 1982.

"L'Europe: auteur politique et puissance mondiale ", in Les futurs de l'Europe, Paris, Le Monde éditions, 1990.

L'économie sociale face à l'ultralibéralisme, Syros, "Pour débattre ", 1994.

Les socialistes français et le mythe révolutionnaire, Hachette Pluriel Référence, 1998.

\section{Articles}

"L'Association française des établissements de crédit banque ", Revue Banque, n 444, 1984.
"Le concept de l'économie sociale », conférence, université de Liège, Crédit coopératif, 1986.

"Les mouvements coopératifs français face à l'échéance de 1992 " (avec Claude Vienney), Lettre du GNC, numéro spécial, 1988.

"La finalité de la coopération dans la banque ", Humanisme et Entreprises, 4/1992.

"Quelques pistes d'innovations coopératives" (en collaboration avec Christophe Raviot), Recma, n² 267, 1998.

"Entre la volonté et la nécessité: témoignage sur l'évolution du Crédit coopératif de 1974 à 1992 ", Recma, n 280, 2001.

"Pour une étude des rapports entre l'Etat et l'économie sociale: spontanéité et instrumentalisation ", Recma, n 284, 2002. 